OPEN ACCESS

Edited by: Francesco Fazio,

University of Messina, Italy

Reviewed by:

Zhen-Yu Du,

East China Normal University, China

Isabel Navarro,

University of Barcelona, Spain

*Correspondence:

Iban Seiliez

iban.seiliez@inra.fr

Specialty section:

This article was submitted to

Aquatic Physiology,

a section of the journal

Frontiers in Physiology

Received: 17 December 2018

Accepted: 28 February 2019

Published: 18 March 2019

Citation:

Séité S, Pioche T, Ory N, Plagnes-Juan E, Panserat S and

Seiliez I (2019) The Autophagic Flux Inhibitor Bafilomycine A1 Affects

the Expression of Intermediary

Metabolism-Related Genes in Trout

Hepatocytes. Front. Physiol. 10:263.

doi: 10.3389/fphys.2019.00263

\section{The Autophagic Flux Inhibitor Bafilomycine A1 Affects the Expression of Intermediary Metabolism-Related Genes in Trout Hepatocytes}

\author{
Sarah Séité ${ }^{1,2,3}$, Tracy Pioche ${ }^{1}$, Nicolas Ory ${ }^{1}$, Elisabeth Plagnes-Juan ${ }^{1}$, \\ Stéphane Panserat ${ }^{1}$ and Iban Seiliez ${ }^{1 *}$ \\ ${ }^{1}$ INRA, E2S UPPA, UMR 1419, Nutrition, Métabolisme, Aquaculture, University of Pau and Pays de l'Adour, \\ Saint-Pée-sur-Nivelle, France, ${ }^{2}$ Evonik Rexim, Ham, France, ${ }^{3}$ Evonik Nutrition and Care GmbH, Hanau, Germany
}

Autophagy is an evolutionarily conserved process of cellular self-eating which emerged these last years as a major adaptive metabolic response to various stresses such as fasting, hypoxia, or environmental pollutants. However, surprisingly very few data is currently available on its role in fish species which are directly exposed to frequent environmental perturbations. Here, we report that the treatment of fasted trout hepatocytes with the autophagy inhibitor Bafilomycine A1 lowered the mRNA levels of many of the gluconeogenesis-related genes and increased those of genes involved in intracellular lipid stores. Concurrently, intracellular free amino acid levels dropped and the expression of the main genes involved in the endoplasmic reticulum (ER) stress exhibited a sharp increase in autophagy inhibited cells. Together these results highlight the strong complexity of the crosstalk between ER, autophagy and metabolism and support the importance of considering this function in future studies on metabolic adaptation of fish to environmental stresses.

\section{Keywords: fish, hepatocyte, autophagy, intermediary metabolism, ER stress, gene expression}

\section{INTRODUCTION}

Macroautophagy (autophagy hereafter) is a cellular function conserved in eukaryotes that allows the recruitment of substrates into lysosomes for their degradation (Bento et al., 2016). In addition to its role as a "cell cleaner," autophagy allows providing energy during fasting or other cellular stress in order to promote survival (Yang and Klionsky, 2010). In mammals, several studies demonstrated that autophagy maintains cellular, and energy homeostasis by degrading and recycling the main

Abbreviations: Acc2, acetyl CoA carboxylase 2; ATF6, activating transcription factor 6; Baf A1, Bafilomycine A1; chop, C/EBP homologous protein; CoA, stearyl coenzyme A; Dgat2, diacylglycerol acetyltransferase 2; edem1, ER degradation enhancing alpha-mannosidase like protein 1; eefla1, eukaryotic elongation factor $1 \alpha 1$; eIF2 $\alpha$, eukaryotic initiation factor $2 \alpha$; fas, fatty acid synthase; fbp, fructose 1,6-bisphosphatase; g6pc, glucose 6-phosphatase; IRE1, inositol requiring 1; pck1, phosphoenol pyruvate carboxykinase 1 (cytosolic); pck2, phosphoenol pyruvate carboxykinase 2 (mitochondrial); PERK, PKR-like ER kinase; plin2, perilipin 2; plin3, perilipin 3; Scd1, stearyl desaturase 1; SIDT2, SID-1 transmembrane family member 2; SREBP, sterol regulatory element-binding proteins; TUBB, $\beta$-tubulin; UPR, unfolded protein response; xbp1, $\mathrm{X}$-box binding protein 1 . 
energy sources (proteins, lipids or glycogen) on exposure to various stresses (Madrigal-Matute and Cuervo, 2016). One of the first metabolic functions attributed to autophagy has been the release of amino acids through protein degradation during starvation. The released amino acids not only sustain protein synthesis under fasting condition, but also feed the tricarboxylic acid cycle for ATP production (Lum et al., 2005; Rabinowitz and White, 2010; Ezaki et al., 2011; Thomas et al., 2018). Furthermore, autophagic proteolysis in liver has been shown to makes a significant contribution to the maintenance of glycaemia during fasting by releasing amino acids for glucose production via gluconeogenesis (Ezaki et al., 2011). In addition to its role in protein breakdown, autophagy has also been shown to play an important role in the degradation of hepatic lipid stores through a selective form of autophagy termed lipophagy (Singh et al., 2009). During this process, autophagy-dependent breakdown of lipid droplets supplies free fatty acids, which undergo $\beta$-oxidation in the mitochondria to support ATP production (Singh et al., 2009; Rambold et al., 2015; Welte, 2015). Although less studied than the two former autophagic processes, lysosomal breakdown of hepatic glycogen might also contribute to glucose homeostasis during some critical periods. In mice, this process known as glycophagy has thus been shown to be necessary to sustain life during the period of postnatal hypoglycemia (Kotoulas et al., 2006). Collectively, these data highlight the critical importance of autophagy for the adaptation of intermediary metabolism to environmental changes.

In fish, this cellular function is attracting growing interest and the number of studies in this field is constantly increasing. As such, induction of autophagy has been demonstrated upon different biotic or abiotic stress situations including pollution (Khangarot, 1992; Chen et al., 2015; Xing et al., 2015), hypoxia (Beck et al., 2016), viral contamination (Liu et al., 2015), fasting, or nutritional imbalance (Seiliez et al., 2012; Yabu et al., 2012; Wei et al., 2017, 2018; Séité et al., 2018; Wang et al., 2018). In these last few years, increasing research also focused on the mechanisms involved in the control of this cellular function in fish, particularly in zebrafish (He et al., 2009; Dowling et al., 2010; He and Klionsky, 2010; Mathai et al., 2017). In contrast, surprisingly, its metabolic role remains poorly explored in these species and very little data is currently available on this subject.

However, we previously reported that rainbow trout treated with the autophagy flux inhibitor agent Colchicine exhibited severe alterations in hepatic carbohydrate and fat metabolisms, as revealed by a significant decrease in plasma glucose levels associated with a decrease of the concentration of some glucogenic amino acids in the liver, but also an increase in hepatic triglyceride and lipid droplet contents (Seiliez et al., 2016). Similarly, recent works provided the evidence for the degradation of lipid stores through lipophagy in the liver of fasted zebrafish (Wang et al., 2018) and yellow catfish (pelteobagrus fulvidraco) fed zinc supplemented diet (Wei et al., 2018). Together, these data are in close agreement with the aforementioned metabolic role of autophagy demonstrated in mammals (Madrigal-Matute and Cuervo, 2016). However, they also suggest that in addition to this previously reported role of autophagy in providing substrates for glucose production, energy furniture, or the synthesis of specific proteins, it could also play a major role in the regulation of the expression of some key metabolic genes. Indeed, these studies pointed out that autophagy inhibited fish exhibited strong perturbations in the mRNA levels of genes involved in hepatic carbohydrate and fat metabolisms (Seiliez et al., 2016; Wang et al., 2018). This would be an unknown function for autophagy. However, they show conflicting outcomes on specific gene expression regulations, precluding a clear picture of the role of autophagy in this process. Such differences could be explained by the divergence of experimental protocols such as the use of (1) different fish models (zebrafish vs. rainbow trout) and (2) different autophagy flux inhibitors (chloroquine vs. colchicine) and deserved further investigations.

In the present study, we treated primary cultures of trout hepatocytes with another autophagy flux inhibitor, the Baf A1, to assess the specificity of the previously reported in vivo effect of colchicine-mediated autophagy inhibition on the expression of several metabolism-related genes in this species. Baf A1 is widely used in vitro as an autophagic flux inhibitor. This drug inhibits the lysosomal V-ATPase to prevent its acidificationas well as the Ca2+ pump SERCA to disrupt autophagosome-lysosome fusion, together resulting in a strong block of autophagic flux (Mauvezin and Neufeld, 2015). The use of primary cultures of trout hepatocytes is an additional asset for our study, as they allow testing the response of the studied factors to specific stimuli independently of their systemic effects. This model is now widely used to improve understanding of intermediary metabolism in fish (Moon et al., 1985).

\section{MATERIALS AND METHODS}

\section{Animals}

Sexually immature rainbow trout having a mean initial weight of $200 \mathrm{~g}$ were obtained from the INRA experimental facilities at Donzacq (Landes, France). Fish were maintained in tank kept in open circuits at a constant water temperature of $17^{\circ} \mathrm{C}$, under natural photoperiod. They were fed to satiety every 2 days with a commercial diet (T-3P classic, Trouw, France). The experiments performed in the present study comply with the EUdirective $2010 / 63 / \mathrm{EU}$ on the protection of animals used for research as well as the decree No 2013-118, 1 February 2013 of the French legislation on the ethical treatment of animals.

\section{Hepatocyte Cell Culture}

Rainbow trout liver cells were isolated from 3 days feed-deprived fish according to the previously detailed protocol (Lansard et al., 2010). We measured the cell viability (>98\%) with trypan blue exclusion method $(0.04 \%$ in $0.15 \mathrm{~mol} / \mathrm{L} \mathrm{NaCl})$ and cells were counted using Neubauer chamber. They were then plated in a 6-well Primaria culture dish (BD) at a density of 3.106 cells/well and incubated at $18^{\circ} \mathrm{C}$, the optimal temperature for cell cultures of trout origin, with complete medium containing modified Hanks' medium (136.9 mmol/L NaCl, $5.4 \mathrm{mmol} / \mathrm{L}$ $\mathrm{KCl}, 0.8 \mathrm{mmol} / \mathrm{L} \mathrm{MgSO}_{4}, 0.44 \mathrm{mmol} / \mathrm{L} \mathrm{KH}_{2} \mathrm{PO}_{4}, 0.33 \mathrm{mmol} / \mathrm{L}$ $\mathrm{Na}_{2} \mathrm{HPO}_{4}, 5 \mathrm{mmol} / \mathrm{L} \mathrm{NaHCO}_{3}$, and $\left.10 \mathrm{mmol} / \mathrm{L} \mathrm{HEPES}\right)$ supplemented with $1 \%$ defatted BSA, $3 \mathrm{mmol} / \mathrm{L}$ glucose, $2 \%$ 
MEM essential amino acid mixture, 1\% MEM non-essential amino acid mixture and $1 \%$ antibiotic antimycotic solution (1X) (sigma). The incubation medium was replaced every $24 \mathrm{~h}$ over the $48 \mathrm{~h}$ of primary cell culture. Microscopic examination ensured that hepatocytes progressively re-associated throughout culture to form cell heap. After 2 days of culture, the cells were incubated in a minimal medium deprived of serum and amino acids (a condition known to activate autophagy) in presence or absence of $100 \mathrm{nM}$ of Baf A1 a concentration commonly used to block autophagosome-lysosome fusion in vitro (Klionsky et al., 2016). Cells were then sampled $4,8,16$, and $24 \mathrm{~h}$ after the treatment and were prepared for western blot analysis or resuspended in TRIZOL reagent (Invitrogen, Carlsbad, CA, United States) and stored at $-80^{\circ} \mathrm{C}$ for subsequent analyses. Each experiment was repeated 2 times.

\section{Protein Extraction and Western Blot Analyses}

Cells were prepared for western blot analyses according to the previously detailed protocol (Lansard et al., 2010). LC3-II levels were measured by western blot as described previously in Belghit et al. (2014) and using the following antibodies: anti-LC3b (\#2775 Cell Signaling Technology) and anti-TUBB (\#2146, Cell Signaling Technology). These antibodies have already been validated in rainbow trout (Belghit et al., 2014).

\section{Quantitative RT-PCR Analyses}

The protocol conditions for sample preparation and quantitative RT-PCR have been previously published (Lansard et al., 2010). The primers used for real time RT-PCR assays are listed in Table 1. Primer of edem 1 and $x b p 1$ were newly designed using Primer3 software. The primers that amplified glucose and lipid metabolism-related genes have already been described in previous studies (Plagnes-Juan et al., 2008; Marandel et al., 2015;

TABLE 1 | Sequences of the primer pairs used in the quantitative real-time RT-PCR assays.

\begin{tabular}{|c|c|c|}
\hline Genes & Forward primer & Reverse primer \\
\hline \multicolumn{3}{|c|}{ Gluconeogenesis related genes } \\
\hline pck1 & ACAGGGTGAGGCAGATGTAGG & CTAGTCTGTGGAGGTCTAAGGGC \\
\hline pck2 & ACAATGAGATGATGTGACTGCA & TGCTCCATCACCTACAACCT \\
\hline fbp1b1 & СTCTCAAGAACCTCTACAGCCT & TCAGTTCTCCCGTTCCCTTC \\
\hline g6pca & GATGGCTTGACGTTCTCCT & AGATCCAGGAGAGTCCTCC \\
\hline g6pcb1 & AGGGACAGTTCGAAAATGGAG & CCAGAGAGGGAAGAAGATGAAGA \\
\hline g6pcb2 & CCTGCGGAACACCTTCTTTG & TCAATTTGTGGCGCTGATGAG \\
\hline \multicolumn{3}{|c|}{ Lipid metabolism related genes } \\
\hline fas & TGATCTGAAGGCCCGTGTCA & GGGTGACGTTGCCGTGGTAT \\
\hline plin2 & CATGGAGTCAGTTGAAGTCGTC & AATTTGTGGCTCCAGCTTGCC \\
\hline plin3 & GATGTCCAACACCGTCACAG & TCGATTTCCAACTCGTCCTC \\
\hline \multicolumn{3}{|c|}{ ER stress related genes } \\
\hline chop & CTGCACACGGTCTGGAGCTG & GGATCTCGTCTGGGATCAGGT \\
\hline edem1 & GAACATCCAAACGGGACAGT & TGAGAAGAGGGAGGGAGTCA \\
\hline xbp1 & CAACCCCGAGAACACAGTTT & AAGTGACACACGCTGTGGTC \\
\hline \multicolumn{3}{|c|}{ Reference gene } \\
\hline eef1a1 & TCCTCTTGGTCGTTTCGCT & ACCCGAGGGACATCCTGTG \\
\hline
\end{tabular}

Seiliez et al., 2016). For the expression analysis, relative quantification of target gene expression was done using the $\Delta$ CT method described by Pfaffl et al. (2002). The relative gene expression value of eefla1 was used for the normalization of the measured expression values of the target mRNA, and was found to not change significantly over sampling time or among treatments (data not shown).

\section{Free Amino Acid Analyses}

Free amino acid concentrations in hepatocytes were determined by ion exchange chromatography with a ninhydrin postcolumn reaction (L-8900 Amino Acid Analyzer, Hitachi HighTechnologies Corporation, Tokyo, Japan).

\section{Statistical Analyses}

Data are expressed as means \pm SD. Normality was assessed using the Shaprio-test, while the equality of variances was determined using Levene's test. When the normality and/or equal variances of data were respected, two-way ANOVA was used to detect significant differences. Following twoway ANOVA analysis, the Tukey test was used for post hoc analysis. For all statistical analyses, the level of significance was set at $P<0.05$.

\section{RESULTS}

\section{Baf A1 Inhibits Autophagy in Trout Hepatocytes}

We first tested the ability of Baf A1 to block autophagy in our cell culture model. For this purpose, we analyzed by western blot the well-established autophagy marker LC3II in cells incubated in a serum- and amino acid-deprived medium (a condition known to activate autophagy) and treated or not with Baf A1 for 4, 8, 16 , and $24 \mathrm{~h}$. During autophagy, LC3 is converted from a nonlipidated cytosolic form (LC3-I) to a phosphatidylethanolamineconjugated form (LC3II) on the autophagosomal membrane (Klionsky et al., 2016). However, LC3-II is also degraded during the late stage of autophagy, and it is now well accepted that the exposure of cells to lysosomal inhibitors, protease inhibitors or agent that block fusion of autophagosome with lysosomes, leads to LC3-II accumulation (Klionsky et al., 2016). As shown in Figure 1, the ratio of LC3-II to TUBB reached significantly higher levels in Baf A1 treated cells compared to non-treated cells. These results indicated that Baf A1 treated cells displayed a loss of autophagy function and that this drug is useful in our cell culture model.

\section{Baf A1 Treatment Affects the Expression of Key Genes of the Intermediary Metabolism in Trout Hepatocytes}

We next addressed the consequences of Baf A1 treatment on the expression of several metabolism-related genes. We first monitored the expression of several genes of the gluconeogenesis in cells incubated in the same conditions described above with or without Baf A1. The obtained results showed that the addition of 

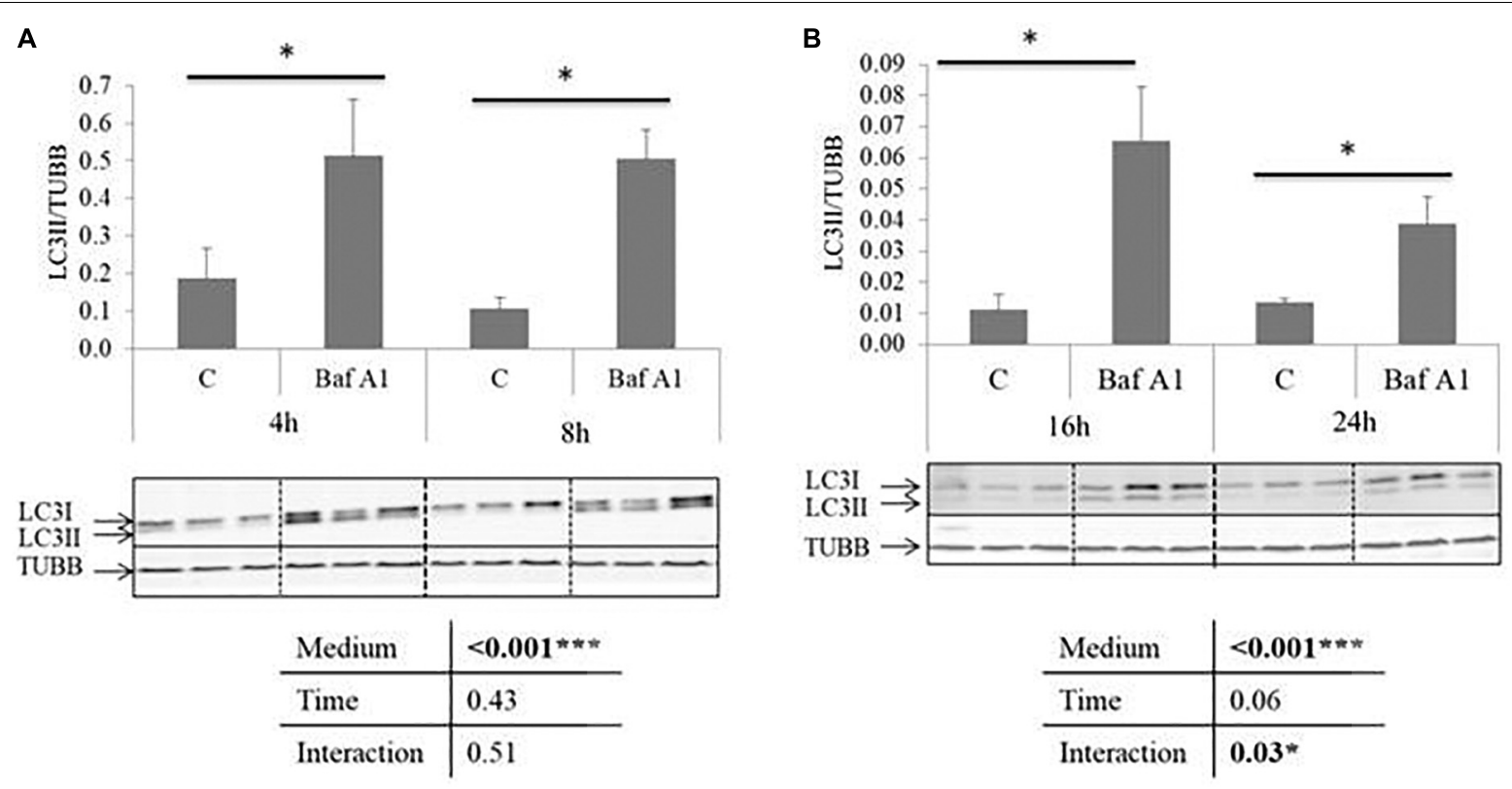

FIGURE 1 | Baf A1 inhibits autophagy in trout hepatocytes. Representative LC3 and TUBB immunoblots of protein homogenates from trout hepatocyte treated with DMSO or Baf A1 for 4, 8. (A) 16 or 24 h (B). Graphs show the ratio between LC3-II and TUBB used as a loading control. Values are means ( $n=6$ ), with standard error of the mean represented by vertical bars. * was used to indicate significant difference between treatment $(P<0.05$; two way anova test).

Baf A1 to the media led to a significant decrease of mRNA levels of gluconeogenesis-related genes $g 6 p c b 1$ and $p c k 1$ regardless of the time of treatment (Figures 2A,E). Similar results were obtained for $g 6 p c a$ and $f b p 1 b 1$ at $24 \mathrm{~h}$ after the treatment (Figures 2C,D) and for pck2 at 16 and $24 \mathrm{~h}$ after the treatment (Figure 2F). In contrast, we observed an increase of mRNA levels of one of the $g 6 p c$ paralogs, the $g 6 p c b 2$, in trout hepatocyte treated with Baf A1 (Figure 2B).

We then analyzed the expression of several genes involved in lipid metabolism. The obtained results showed that mRNA levels of fas increased in cells treated with Baf A1 (Figure 3A). Similar results were obtained for plin2 and plin3, two critical regulators of hepatic neutral lipid storage (Figures 3B,C).

Overall, these data confirmed our previous in vivo results obtained with Colchicine and established a tight link between the activity of autophagy and the expression of several glucose and lipid metabolism-related genes.

\section{Baf A1 Treatment Lowers the Level of Free Amino Acids in Trout Hepatocytes}

It is now well established that the expression of many metabolism-related genes is under the tight control of amino acid availability (Lansard et al., 2010, 2011). Autophagy being one of the main systems for the release of free amino acids during fasting, we wondered whether the effects of Baf A1 on metabolic gene expression could be related to a decrease in free amino acid levels in hepatocytes whose autophagy has been inhibited. We therefore, monitored the concentration of the main amino acids in fasted cells treated or not with Baf A1. As shown in Figure 4, hepatocytes treated with Baf A1 exhibited lower levels of most of the analyzed amino acids, in accordance with the reported role of liver autophagy on amino acid release during starvation. This global decrease in amino acid release in hepatocytes treated with BafA1 could therefore contribute to perturb the expression of the studied genes.

\section{Baf A1 Treatment Leads to ER Stress}

Another hypothesis to explain the effect of Baf A1 on the expression of the studied genes concerns the endoplasmic reticulum (ER) stress. Accumulating evidences demonstrated that autophagy dysregulation causes ER stress (Yang et al., 2010), which has been shown to strongly impact the expression of intermediary metabolism-related genes (Lee et al., 2012; Wang and Kaufman, 2014; Zhou and Liu, 2014). However, to our knowledge, few if no data is available on the effect of autophagy dysregulation-mediated ER stress on the expression of intermediary metabolism-related genes. In the present study, we therefore sought to determine whether Baf A1 caused ER stress in our cells. To this end, we analyzed, in fasted hepatocytes treated with or without Baf A1, the expression of three target genes chop, $x b p 1$, and edem1 of the main ER-stress sensing pathways PERK, ATF6, and IRE1 pathways, respectively. As shown in Figure 5A, the mRNA levels of chop significantly increased in Baf A1 treated cells in comparison to control cells 4,8 and $16 \mathrm{~h}$ after the treatment. Similar results were obtained for $x b p 1$, with an increase at 4 and $8 \mathrm{~h}$ after the treatment (Figure 5B). Likewise, the mRNA levels of edem 1 increased $24 \mathrm{~h}$ after the treatment (Figure 5C), in line with previous findings demonstrating that edem 1 is a late ER-stress marker. Overall, the results obtained clearly show that hepatocytes treated with Baf A1 display sign of ER stress, which in turn could affect the expression of the studied genes. 
A

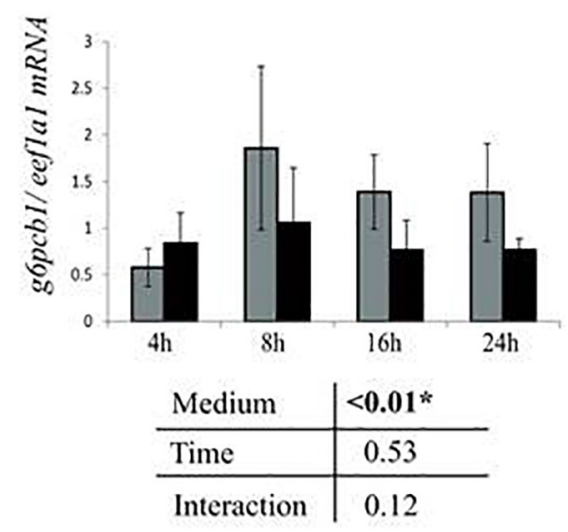

C

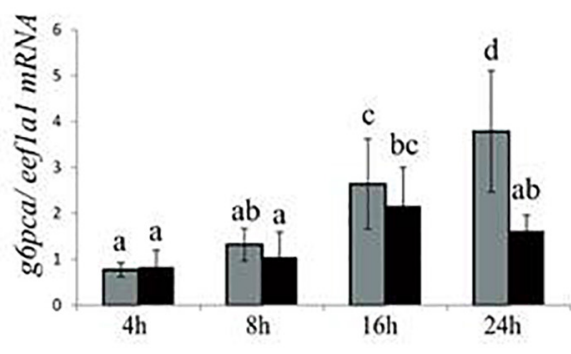

\begin{tabular}{l|l} 
Medium & $<\mathbf{0 . 0 1 *}$ \\
\hline Time & $<0.0001^{* * *}$ \\
\hline Interaction & $<0.0001^{* * *}$
\end{tabular}

E

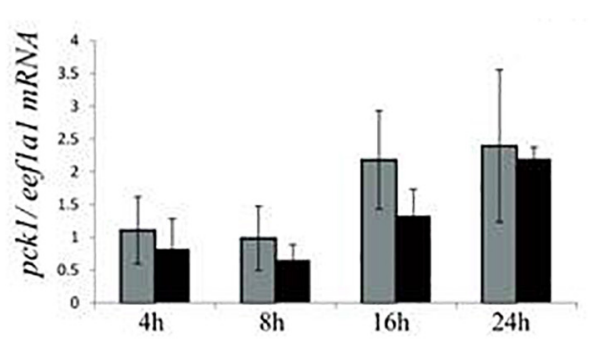

\begin{tabular}{l|l} 
Medium & $<0.01^{*}$ \\
\hline Time & $<0.0001^{* * *}$ \\
\hline Interaction & 0.85
\end{tabular}

B

$\square \mathrm{C}$

Baf Al

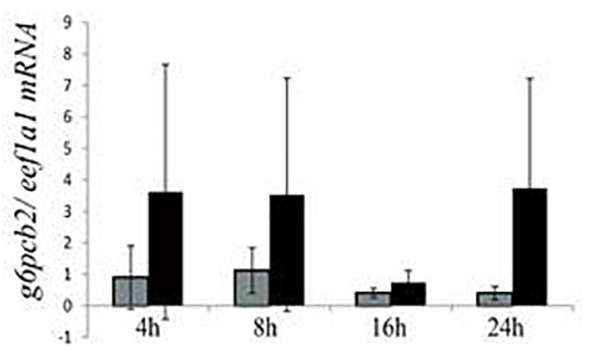

\begin{tabular}{l|l} 
Medium & $<\mathbf{0 . 0 0 1}{ }^{* *}$ \\
\hline Time & 0.35 \\
\hline Interaction & $\mathbf{0 . 8 8}$
\end{tabular}

D

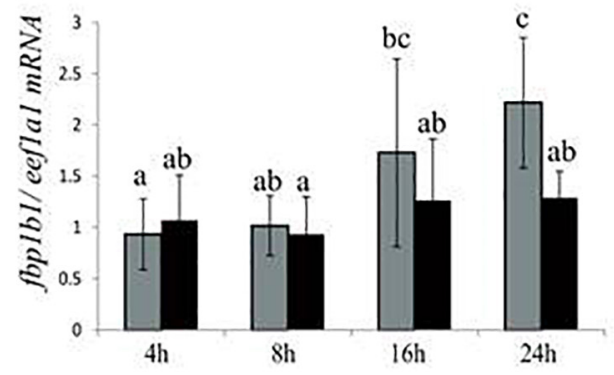

\begin{tabular}{l|l} 
Medium & $<0.03^{*}$ \\
\hline Time & $<0.0001^{* * *}$ \\
\hline Interaction & $<0.001^{* *}$
\end{tabular}

$\mathbf{F}$

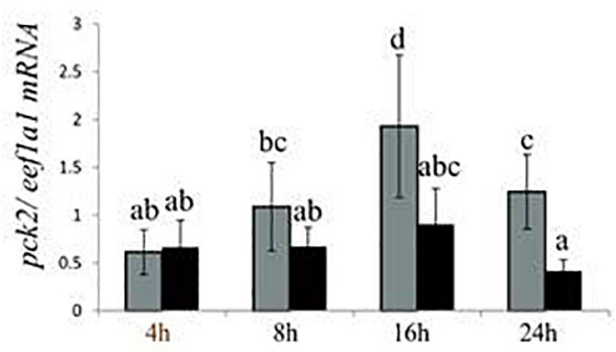

\begin{tabular}{l|c} 
Medium & $<\mathbf{0 . 0 0 0 1 * * *}$ \\
\hline Time & 0.056 \\
\hline Interaction & $<0.001^{* *}$
\end{tabular}

FIGURE 2 | Baf A1 treatment affects mRNA levels of gluconeogenic genes. Hepatocytes were treated with DMSO or Baf A1 for 4, 8, 16, or 24 h. Hepatocyte mRNA levels of (A) g6pcb1, (B) g6pcb2, (C) G6pca, (D) fbp1b, (E) pck1, and (F) pck2 were measured using quantitative real time RT-PCR assays. Expression values are normalized with the eukaryotic translation elongation factor $1 \alpha 1$ (eef1a1) mRNA. Value are means $(n=6)$ with standard error represented by vertical bars and were analyzed using two-way ANOVA $(P<0.05)$, followed by Tukey's post hoc test for multiple comparisons. When interaction between sampling time and treatment is significant, lowercases letters ( $a, b, c$, and d) represent statistically significant differences ( $P<0.05$, Tukey's HSD). 


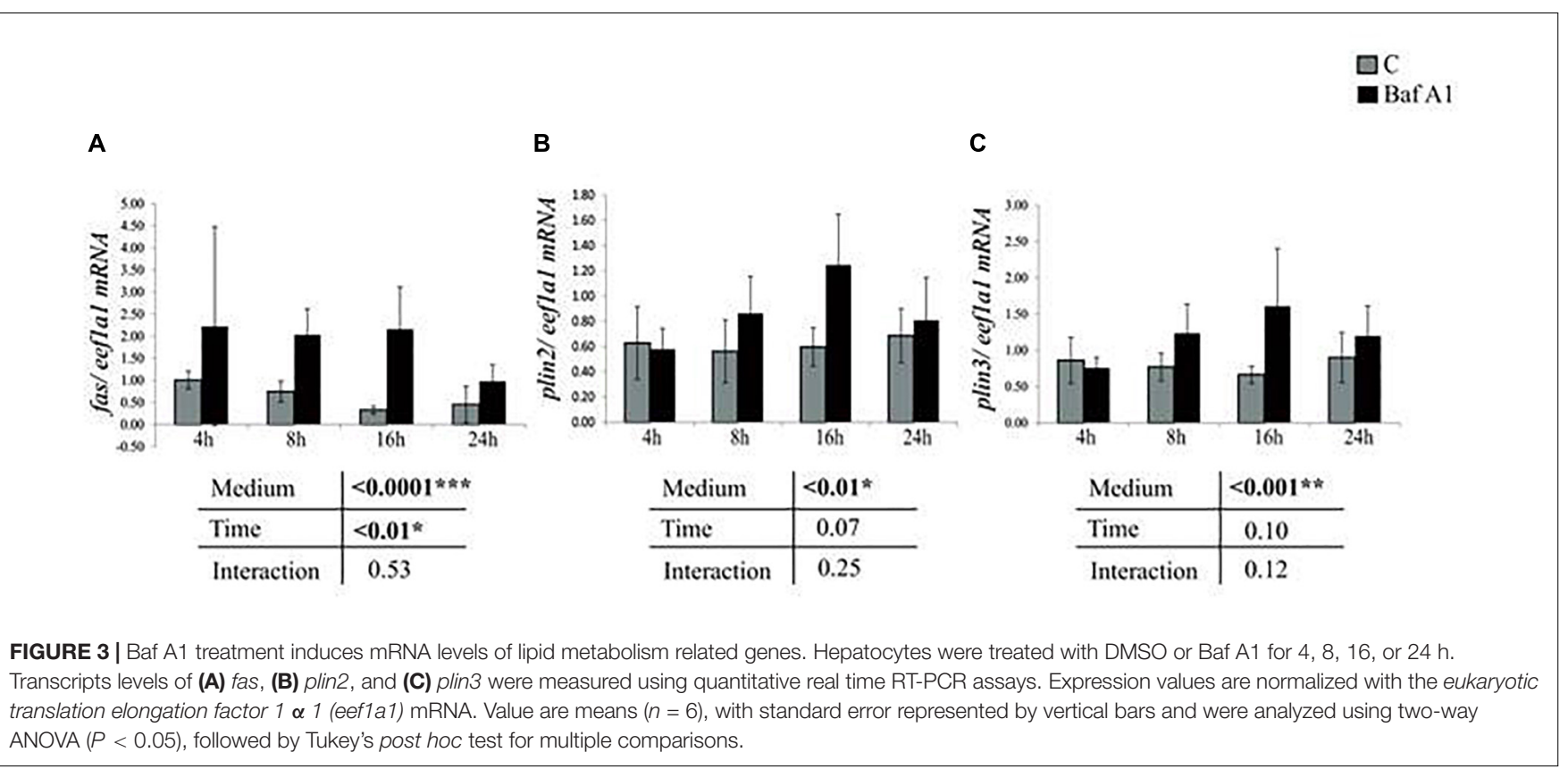

\section{DISCUSSION}

Autophagy has long been considered merely as a cellular waste disposal and recycling mechanism. However, studies in recent years have highlighted its major role for the adaptation of metabolism to environmental changes (Kroemer et al., 2010; Chen et al., 2015; Chiarelli et al., 2016; Tang, 2016). In this regard, we and others showed that treatment of fasted fish (rainbow trout or zebrafish) with autophagy flux inhibitor agents (colchicine or chloroquine) led to strong defaults in intracellular substrates delivery for glucose production or energy furniture (Seiliez et al., 2016; Wang et al., 2018). Interestingly, these studies also pointed out sever perturbations in the mRNA levels of several intermediary metabolism-related genes in these fish, establishing a new potential link between autophagy and intermediary metabolism. However, probably due to divergences of experimental protocols (including the species investigated and the used autophagy flux inhibitors), these studies led to conflicting results with respect to the regulations of specific gene expression, precluding a clear picture of the role of autophagy in this process.

In the present study, we demonstrated that Baf A1 treatment of trout hepatocytes decreased the mRNA levels of genes involved in gluconeogenesis and conversely, increased those of genes involved in lipogenesis and lipid storage. Although it is well accepted that Baf A1 is an autophagy inhibitor, it may also have other side effects. For instance, some data reported that it has some effects on mitochondria quality (Yuan et al., 2015; Redmann et al., 2017), making it difficult to determine which effects on metabolism-related mRNAs could be a consequence of inhibiting autophagy or of direct effects on mitochondria independently of autophagy. However, our results are in close agreement with those previously reported in trout showing that in vivo treatment with colchicine (which act on autophagy by inducing microtubule disassembly) led to a similar lowering effect on the mRNA levels of gluconeogenesis-related genes and an increasing effect on both plin2 and plin3 (Seiliez et al., 2016), suggesting that the observed effects are specific to autophagy inhibition. Interestingly, previous findings in mammals also evidenced a tight link between the activity of autophagy and mRNA levels of some enzymes involved in glucose metabolism (Wang et al., 2015). Acute suppression of autophagy with lysosome inhibitors (Chloroquine or Bafilomycin A1) in statin treated human liver cancer cell line (HepG2 cells) has thus been shown to reduce mRNA levels of the two gluconeogenic enzymes $g 6 p c$ and $p c k 1$ (Wang et al., 2015). Similarly, the statininduced increase in expression of $g 6 p c$ and $p c k 1$ was blocked in Atg7-deficient hepatocytes, providing a genetic confirmation of these results (Wang et al., 2015). However, another study suggests the opposite role of autophagy in gluconeogenesis with the finding that overexpression of Atg7 reduces mRNA levels of $g 6 p c$ and $p c k 1$ in the livers of mice (Yang et al., 2010); But the induction of autophagy by Atg7 overexpression was not verified in this study, preventing to conclude on the specific role of this function in the observed effects. More recently, Wang et al. (2018) showed that chloroquine treatment of fasted zebrafish inhibited the hepatic expression of most genes related to lipid metabolism and conversely upregulated those of carbohydrates metabolism, making possible the existence of species-dependent effects of autophagy inhibition. Overall, these data support a close link between autophagy and the mRNA levels of metabolic genes, although the exact nature of this relationship, which likely depends on many factors (including the species studied and/or the protocol used to monitor this link), remains to be clarified.

It is now clearly established that the expression of a wide range of hepatic genes involved in the intermediary metabolism is under the control of amino acid availability. In trout hepatocytes, free amino acid addition to an amino acid-deprived medium 


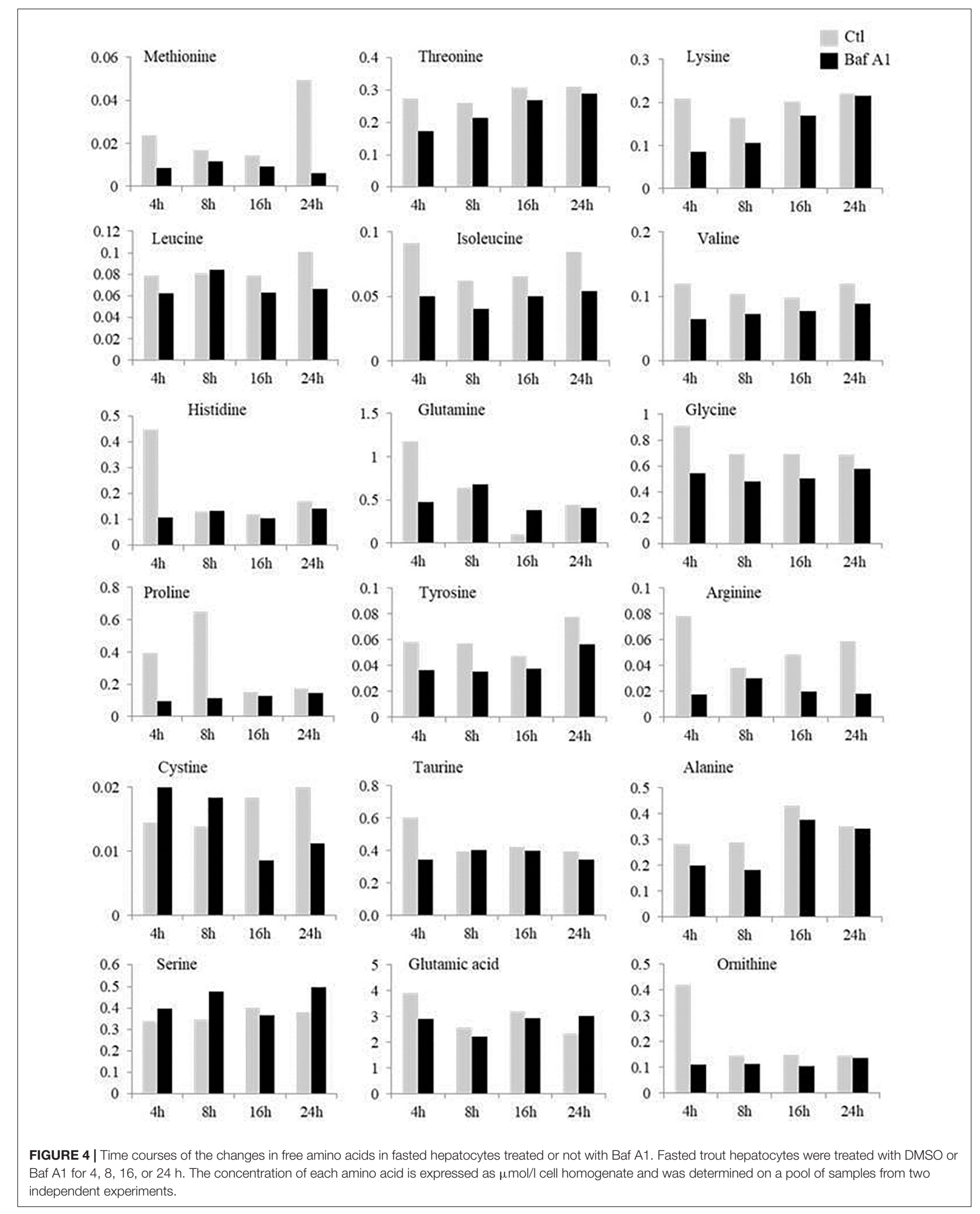



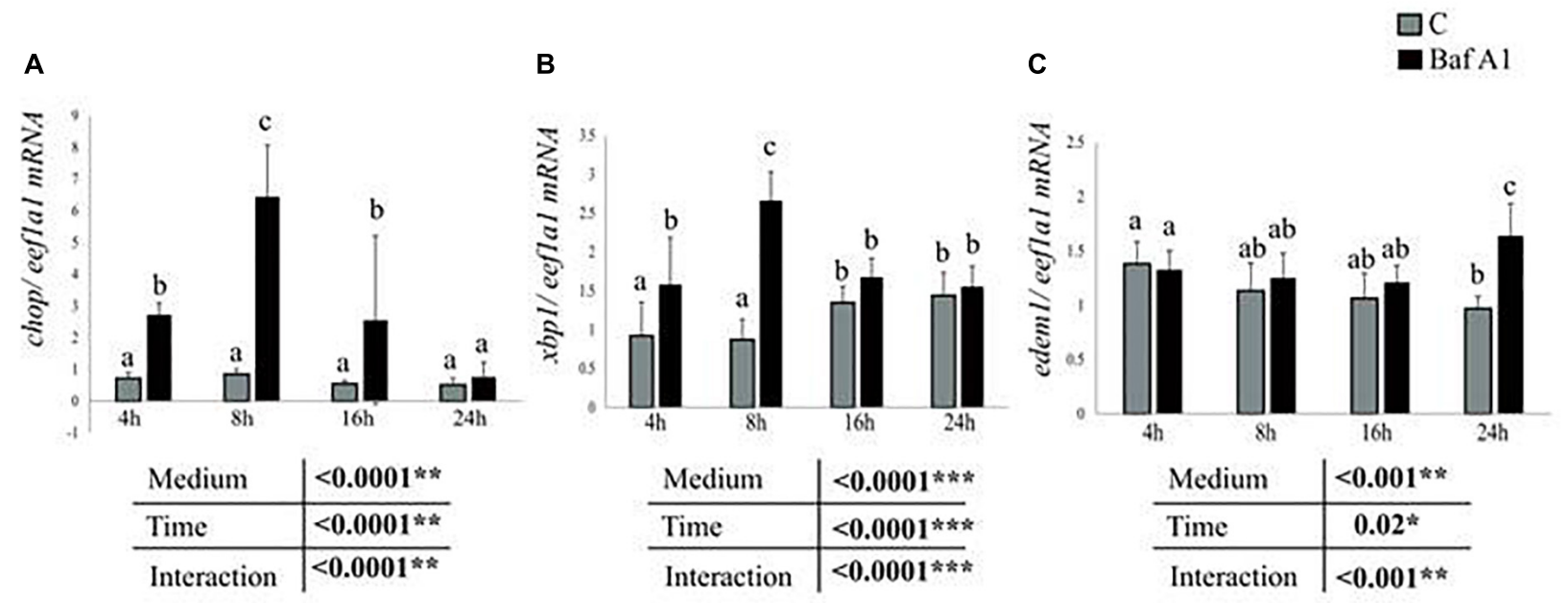

FIGURE 5 | Baf A1 treatment induces ER stress markers. Hepatocytes were treated with DMSO or Baf A1 for 4, 8, 16, or 24 h. Hepatocyte mRNA levels of (A), chop (B), xbp1, and (C) edem1 were measured using quantitative real time RT-PCR assays. Expression values are normalized with the eukaryotic translation elongation factor $1 \propto 1$ (eef1a1) mRNA. Value are means $(n=6)$, with standard error with represented by vertical bars and were analyzed using two-way ANOVA $(P<0.05)$, followed by Tukey's post hoc test for multiple comparisons. When interaction between sampling time and treatment is significant, lowercases letters (a, b, and c) represent statistically significant differences $(P<0.05$, Tukey's HSD).

has thus been shown to up-regulate the mRNA levels of gluconeogenesis-related enzymes (Lansard et al., 2010, 2011). As autophagy is described as one of the main amino acid provider during fasting (Kuma et al., 2004; Ezaki et al., 2011), we therefore hypothesized that the observed effect of Baf A1 treatment on the mRNA levels of metabolic genes could be due to a default in free amino acid release in autophagy-inhibited hepatocytes. Accordingly, Baf A1 treated cells exhibited lower levels of most of the analyzed amino acids compared to control cells. Such an effect of autophagy in providing amino acids endogenously to sustain mechanistic target of rapamycin complex 1 (mTORC1) signaling when extracellular amino acids are limited has previously been reported in C2C12 murine myotubes (Yu and Long, 2014), and could therefore be also at play in the observed effect of Baf A1 on the studied genes. Interestingly, the expression of $g 6 p c b 2$ which, in contrast to the other analyzed gluconeogenesis-related genes increased in Baf A1 treated hepatocytes, was previously shown to exhibit an opposite regulation by feeding different levels of proteins and by amino acids levels in hepatocytes compared to other $g 6 p c$ paralogs (Marandel et al., 2015; Lucie et al., 2016), tipping the scale in favor of a default of autophagy-dependent release of amino acids in the observed Baf A1 effect. However, not all studied genes are known to be under the control of amino acids per se. This is particularly the case for the gene fas, whose expression has already been shown to be not directly affected by the addition of amino acids to an amino acid-free medium in trout hepatocytes (Lansard et al., 2010, 2011). Instead, it is possible that the induction of ER stress observed in autophagyinhibited hepatocytes plays an important role in the observed effect of Baf A1 treatment on these genes. Indeed, previous studies have shown that ER stress plays a critical role in regulation of lipid metabolism (Sriburi et al., 2004; Bobrovnikova-Marjon et al., 2008; Oyadomari et al., 2008; Rutkowski et al., 2008; Kammoun et al., 2009; Zhang et al., 2014). According to these studies, ER stress leads to activation of the evolutionarily conserved UPR signaling system in order to restore ER homeostasis (Shen et al., 2004). Accumulating evidence shows that activation of the UPR pathways can modulate lipid metabolism by controlling the transcriptional regulation of lipogenesis and triglyceride storage (Basseri and Austin, 2012; Han and Kaufman, 2016). For example, PERK and eIF2 $\alpha$ phosphorylation are induced by antipsychotic drugs, resulting in increased lipid accumulation in hepatocytes through activation of sterol regulatory elementbinding proteins SREBP-1c and SREBP-2, two transcription factors that regulate the expression of critical enzymes involved in lipogenic pathways including fas (Gosmain et al., 2005; Lauressergues et al., 2012). XBP1 also seems to be involved in the lipid metabolism through both direct and indirect activation of the transcription of key lipogenic genes in the liver, including fas, plin2 as well as CoA, desaturase 1 ( $S c d 1)$, Dgat2, and Acc2 (Lee et al., 2008, 2012). Together, these data support a possible role of ER stress in the observed effect of Baf A1 on the mRNA levels of enzymes involved in lipid metabolism. Noteworthy, UPR signaling has also been shown to affect the expression of genes involved in glucose metabolism and more particularly those of the gluconeogenesis pathway (Wagner and Moore, 2011).

Finally, recent findings in mammals reported a novel RNA degradation system called RNautophagy, during which direct import of RNA into lysosomes followed by degradation takes place (Fujiwara et al., 2013). During this process, the putative nucleic acid transporter SIDT2 predominantly localizes to lysosomes and mediates the translocation of RNA into lysosomes (Aizawa et al., 2016; Contu et al., 2017). Interestingly, the authors found that treatment of cells with lysosome inhibitors (chloroquine or Bafilomycin A1) hindered the SIDT2 overexpression-mediated increase in intracellular 
RNA degradation. These data make therefore the impairment of RNautophagy a possible mechanism of the observed inducing effect of Baf A1 in the level of some mRNAs. However, it remains to be established whether or not RNautophagy or a RNautophagy-like process exists in fish.

\section{Wrapping-Up}

In the present study, we report that the treatment of fasted trout hepatocytes with Baf A1 strongly perturb the mRNA expression of several genes involved in glucose and lipid metabolisms. These results are in close agreement with those already reported with other autophagy inhibitors both in mammals and fish, and support a tight link between autophagy activity and the mRNA levels of metabolic genes. The underlying mechanisms are likely multiple and highlight the complexity of the crosstalk between ER, autophagy and metabolism.

Interestingly, the observed decrease in mRNA levels of gluconeogenic genes in cells treated with Baf A1 is also consistent with the reported role of autophagy in the maintenance of blood glucose during fasting by releasing amino acids for glucose production via gluconeogenesis (Ezaki et al., 2011). Similarly, we observed an increase in mRNA levels of FAS and the two LD-associated proteins PLIN2 and PLIN3 in Baf A1 treated cells in agreement with the wellestablished role of autophagy in the control of lipid stores during fasting (Singh et al., 2009; Wang et al., 2018; Wei et al., 2018). Autophagy could thus combine its role as a supplier of substrates for the production of glucose or energy furniture with the molecular regulation of several related metabolic enzymes.

\section{REFERENCES}

Aizawa, S., Fujiwara, Y., Contu, V. R., Hase, K., Takahashi, M., Kikuchi, H., et al. (2016). Lysosomal putative RNA transporter SIDT2 mediates direct uptake of RNA by lysosomes. Autophagy 12, 565-578. doi: 10.1080/15548627.2016. 1145325

Basseri, S., and Austin, R. C. (2012). Endoplasmic reticulum stress and lipid metabolism: mechanisms and therapeutic potential. Biochem. Res. Int. 2012:841362. doi: 10.1155/2012/841362

Beck, B. H., Fuller, S. A., Li, C., Green, B. W., Zhao, H., Rawles, S. D., et al. (2016). Hepatic transcriptomic and metabolic responses of hybrid striped bass (Morone saxatilis $\times$ Morone chrysops) to acute and chronic hypoxic insult. Comp. Biochem. Physiol. Part D Genomics Proteomics 18, 1-9. doi: 10.1016/j. cbd.2016.01.005

Belghit, I., Skiba-Cassy, S., Geurden, I., Dias, K., Surget, A., Kaushik, S., et al. (2014). Dietary methionine availability affects the main factors involved in muscle protein turnover in rainbow trout (Oncorhynchus mykiss). Br. J. Nutr. 112, 493-503. doi: 10.1017/S0007114514001226

Bento, C. F., Renna, M., Ghislat, G., Puri, C., Ashkenazi, A., Vicinanza, M., et al. (2016). Mammalian autophagy: how does it work? Annu. Rev. Biochem. 85, 685-713. doi: 10.1146/annurev-biochem-060815-014556

Bobrovnikova-Marjon, E., Hatzivassiliou, G., Grigoriadou, C., Romero, M., Cavener, D. R., Thompson, C. B., et al. (2008). PERK-dependent regulation of lipogenesis during mouse mammary gland development and adipocyte differentiation. Proc. Natl. Acad. Sci. U.S.A. 105, 16314-16319. doi: 10.1073/ pnas.0808517105

Chen, D., Zhang, Z., Yao, H., Liang, Y., Xing, H., and Xu, S. (2015). Effects of atrazine and chlorpyrifos on oxidative stress-induced autophagy in the immune
In the future, important issues will be to confirm these observations by establishing fish cell lines whose autophagy is genetically invalidated, which is now possible with the CRISPRCas9 technology. Gaining knowledge in the relationships between ER, autophagy and metabolism is of paramount for a better understanding of the mechanisms involved in metabolic adaptation of fish to environmental stresses.

\section{AUTHOR CONTRIBUTIONS}

SS, SP, and IS designed the research. TP, NO, and EP-J conducted the analyses. All the data were obtained and analyzed by TP, NO, and SS under the supervision of SP and IS. The manuscript was written by SS and critically revised by IS and SP. All authors have read and approved the final manuscript. IS had primary responsibility for final content.

\section{ACKNOWLEDGMENTS}

The authors acknowledge Evonik Industries and Agence National de la Recherche et de la Technologie (ANRT, France) for the scholarship to SS (CIFRE Ph.D. Research Grant). Special thanks are due to K. Dias (INRA-UPPA, UMR1419 Nutrition Métabolisme Aquaculture, F-64310 Saint-Pée-surNivelle, France) and N. Hafnaoui (INRA, UMR1019 UNH, CRNH Auvergne, F-63000 Clermont-Ferrand, France) for their technical assistance. The authors also thank the staff at the fish farm (F. Vallée, F. Terrier, A. Lanuque, F. Sandres, and P. Aguirre) for animal care.

organs of common carp (Cyprinus carpio L.). Fish Shellfish Immunol. 44, 12-20. doi: 10.1016/j.fsi.2015.01.014

Chiarelli, R., Martino, C., Agnello, M., Bosco, L., and Roccheri, M. C. (2016). Autophagy as a defense strategy against stress: focus on Paracentrotus lividus sea urchin embryos exposed to cadmium. Cell Stress Chaperones 21, 19-27. doi: 10.1007/s12192-015-0639-3

Contu, V. R., Hase, K., Kozuka-Hata, H., Oyama, M., Fujiwara, Y., Kabuta, C., et al. (2017). Lysosomal targeting of SIDT2 via multiple Yxx $\Phi$ motifs is required for SIDT2 function in the process of RNautophagy. J. Cell Sci. 130, 2843-2853. doi: $10.1242 /$ jcs. 202481

Dowling, J. J., Low, S. E., Busta, A. S., and Feldman, E. L. (2010). Zebrafish MTMR14 is required for excitation-contraction coupling, developmental motor function and the regulation of autophagy. Hum. Mol. Genet. 19, 26682681. doi: 10.1093/hmg/ddq153

Ezaki, J., Matsumoto, N., Takeda-Ezaki, M., Komatsu, M., Takahashi, K., Hiraoka, Y., et al. (2011). Liver autophagy contributes to the maintenance of blood glucose and amino acid levels. Autophagy 7, 727-736. doi: 10.4161/auto. 7.7.15371

Fujiwara, Y., Furuta, A., Kikuchi, H., Aizawa, S., Hatanaka, Y., Konya, C., et al. (2013). Discovery of a novel type of autophagy targeting RNA. Autophagy 9, 403-409. doi: 10.4161/auto.23002

Gosmain, Y., Dif, N., Berbe, V., Loizon, E., Rieusset, J., Vidal, H., et al. (2005). Regulation of SREBP-1 expression and transcriptional action on HKII and FAS genes during fasting and refeeding in rat tissues. J. Lipid Res. 46, 697-705. doi: 10.1194/jlr.M400261-JLR200

Han, J., and Kaufman, R. J. (2016). The role of ER stress in lipid metabolism and lipotoxicity. J. Lipid Res. 57, 1329-1338. doi: 10.1194/jlr.R06 7595 
He, C., Bartholomew, C. R., Zhou, W., and Klionsky, D. J. (2009). Assaying autophagic activity in transgenic GFP-Lc3 and GFP-Gabarap zebrafish embryos. Autophagy 5, 520-526. doi: 10.4161/auto.5.4.7768

He, C., and Klionsky, D. J. (2010). Analyzing autophagy in zebrafish. Autophagy 6, 642-644. doi: 10.4161/auto.6.5.12092

Kammoun, H. L., Chabanon, H., Hainault, I., Luquet, S., Magnan, C., Koike, T., et al. (2009). GRP78 expression inhibits insulin and ER stress-induced SREBP$1 \mathrm{c}$ activation and reduces hepatic steatosis in mice. J. Clin. Invest. 119, 12011215. doi: 10.1172/JCI37007

Khangarot, B. S. (1992). Copper-induced hepatic ultrastructural alterations in the snake-headed fish, Channa punctatus. Ecotoxicol. Environ. Safety 23, 282-293. doi: 10.1016/0147-6513(92)90078-H

Klionsky, D. J., Abdelmohsen, K., Abe, A., Abedin, M. J., Abeliovich, H., Acevedo Arozena, A., et al. (2016). Guidelines for the use and interpretation of assays for monitoring autophagy. Autophagy 12, 1-222. doi: 10.1080/15548627.2015. 1100356

Kotoulas, O. B., Kalamidas, S. A., and Kondomerkos, D. J. (2006). Glycogen autophagy in glucose homeostasis. Pathol. Res. Pract. 202, 631-638. doi: 10. 1016/j.prp.2006.04.001

Kroemer, G., Mariño, G., and Levine, B. (2010). Autophagy and the integrated stress response. Mol. Cell. 40, 280-293. doi: 10.1016/j.molcel.2010.09.023

Kuma, A., Hatano, M., Matsui, M., Yamamoto, A., Nakaya, H., Yoshimori, T., et al. (2004). The role of autophagy during the early neonatal starvation period. Nature 432, 1032-1036. doi: 10.1038/nature03029

Lansard, M., Panserat, S., Plagnes-Juan, E., Dias, K., Seiliez, I., and Skiba-Cassy, S. (2011). l-Leucine, l-methionine, and 1-lysine are involved in the regulation of intermediary metabolism-related gene expression in rainbow trout hepatocytes. J. Nutr. 141, 75-80. doi: 10.3945/jn.110.124511

Lansard, M., Panserat, S., Plagnes-Juan, E., Seiliez, I., and Skiba-Cassy, S. (2010). Integration of insulin and amino acid signals that regulate hepatic metabolismrelated gene expression in rainbow trout: role of TOR. Amino Acids. 39, 801-810. doi: 10.1007/s00726-010-0533-3

Lauressergues, E., Bert, E., Duriez, P., Hum, D., Majd, Z., Staels, B., et al. (2012). Does endoplasmic reticulum stress participate in APD-induced hepatic metabolic dysregulation? Neuropharmacology 62, 784-796. doi: 10.1016/j. neuropharm.2011.08.048

Lee, A.-H., Scapa, E. F., Cohen, D. E., and Glimcher, L. H. (2008). Regulation of hepatic lipogenesis by the transcription factor XBP1. Science 320, 1492-1496. doi: $10.1126 /$ science. 1158042

Lee, J.-S., Mendez, R., Heng, H. H., Yang, Z., and Zhang, K. (2012). Pharmacological ER stress promotes hepatic lipogenesis and lipid droplet formation. Am. J. Transl. Res. 4, 102-113.

Liu, C., Zhao, Y., Chen, L., Zhang, Z., Li, M., and Li, S. (2015). Avermectin induced autophagy in pigeon spleen tissues. Chemico Biol. Interact. 242, 327-333. doi: 10.1016/j.cbi.2015.10.022

Lucie, M., Weiwei, D., Stéphane, P., and Sandrine, S.-C. (2016). The five glucose6-phosphatase paralogous genes are differentially regulated by insulin alone or combined with high level of amino acids and/or glucose in trout hepatocytes. Mol. Biol. Rep. 43, 207-211. doi: 10.1007/s11033-016-3962-6

Lum, J. J., DeBerardinis, R. J., and Thompson, C. B. (2005). Autophagy in metazoans: cell survival in the land of plenty. Nat. Rev. Mol. Cell Biol. 6, 439-448. doi: 10.1038/nrm 1660

Madrigal-Matute, J., and Cuervo, A. M. (2016). Regulation of liver metabolism by autophagy. Gastroenterology 150, 328-339. doi: 10.1053/j.gastro.2015.09.042

Marandel, L., Seiliez, I., Véron, V., Skiba-Cassy, S., and Panserat, S. (2015). New insights into the nutritional regulation of gluconeogenesis in carnivorous rainbow trout (Oncorhynchus mykiss): a gene duplication trail. Physiol. Genomics 47, 253-263. doi: 10.1152/physiolgenomics.00026.2015

Mathai, B. J., Meijer, A. H., and Simonsen, A. (2017). Studying autophagy in zebrafish. Cells 6:21. doi: 10.3390/cells6030021

Mauvezin, C., and Neufeld, T. P. (2015). Bafilomycin A1 disrupts autophagic flux by inhibiting both V-ATPase-dependent acidification and Ca-P60A/SERCAdependent autophagosome-lysosome fusion. Autophagy 11, 1437-1438. doi: 10.1080/15548627.2015.1066957

Moon, T. W., Walsh, P. J., and Mommsen, T. P. (1985). Fish hepatocytes: a model metabolic system. Can. J. Fish. Aquat. Sci. 42, 1772-1782. doi: 10.1139/f85-222

Oyadomari, S., Harding, H. P., Zhang, Y., Oyadomari, M., and Ron, D. (2008). Dephosphorylation of translation initiation factor $2 \alpha$ enhances glucose tolerance and attenuates hepatosteatosis in mice. Cell Metab. 7, 520-532. doi: 10.1016/j.cmet.2008.04.011

Pfaffl, M. W., Horgan, G. W., and Dempfle, L. (2002). Relative expression software tool (REST) for group-wise comparison and statistical analysis of relative expression results in real-time PCR. Nucleic Acids Res. 30:e36. doi: 10.1093/nar/ 30.9.e36

Plagnes-Juan, E., Lansard, M., Seiliez, I., Médale, F., Corraze, G., Kaushik, S., et al. (2008). Insulin regulates the expression of several metabolism-related genes in the liver and primary hepatocytes of rainbow trout (Oncorhynchus mykiss). J. Exp. Biol. 211, 2510-2518. doi: 10.1242/jeb.018374

Rabinowitz, J. D., and White, E. (2010). Autophagy and metabolism. Science 330, 1344-1348. doi: 10.1126/science.1193497

Rambold, A. S., Cohen, S., and Lippincott-Schwartz, J. (2015). Fatty acid trafficking in starved cells: regulation by lipid droplet lipolysis, autophagy, and mitochondrial fusion dynamics. Dev. Cell 32, 678-692. doi: 10.1016/j.devcel. 2015.01.029

Redmann, M., Benavides, G. A., Berryhill, T. F., Wani, W. Y., Ouyang, X., Johnson, M. S., et al. (2017). Inhibition of autophagy with bafilomycin and chloroquine decreases mitochondrial quality and bioenergetic function in primary neurons. Redox Biol. 11, 73-81. doi: 10.1016/j.redox.2016.11.004

Rutkowski, D. T., Wu, J., Back, S.-H., Callaghan, M. U., Ferris, S. P., Iqbal, J., et al. (2008). UPR pathways combine to prevent hepatic steatosis caused by ER stress-mediated suppression of transcriptional master regulators. Dev. Cell 15, 829-840. doi: 10.1016/j.devcel.2008.10.015

Seiliez, I., Belghit, I., Gao, Y., Skiba-Cassy, S., Dias, K., Cluzeaud, M., et al. (2016). Looking at the metabolic consequences of the colchicine-based in vivo autophagic flux assay. Autophagy 12, 343-356. doi: 10.1080/15548627.2015. 1117732

Seiliez, I., Gabillard, J.-C., Riflade, M., Sadoul, B., Dias, K., Avérous, J., et al. (2012). Amino acids downregulate the expression of several autophagy-related genes in rainbow trout myoblasts. Autophagy 8, 364-375. doi: 10.4161/auto. 18863

Séité, S., Mourier, A., Camougrand, N., Salin, B., Figueiredo-Silva, A. C., FontagnéDicharry, S., et al. (2018). Dietary methionine deficiency affects oxidative status, mitochondrial integrity and mitophagy in the liver of rainbow trout (Oncorhynchus mykiss). Sci. Rep. 8:10151. doi: 10.1038/s41598-018-28559-8

Shen, X., Zhang, K., and Kaufman, R. J. (2004). The unfolded protein response-a stress signaling pathway of the endoplasmic reticulum. J. Chem. Neuroanat. 28, 79-92. doi: 10.1016/j.jchemneu.2004.02.006

Singh, R., Kaushik, S., Wang, Y., Xiang, Y., Novak, I., Komatsu, M., et al. (2009). Autophagy regulates lipid metabolism. Nature 458, 1131-1135. doi: 10.1038/ nature07976

Sriburi, R., Jackowski, S., Mori, K., and Brewer, J. W. (2004). XBP1: a link between the unfolded protein response, lipid biosynthesis, and biogenesis of the endoplasmic reticulum. J. Cell Biol. 167, 35-41. doi: 10.1083/jcb.200406136

Tang, B. L. (2016). Autophagy in response to environmental stresses: new monitoring perspectives. Ecol. Indic. 60, 453-459. doi: 10.1016/j.ecolind.2015. 07.022

Thomas, M., Davis, T., Loos, B., Sishi, B., Huisamen, B., Strijdom, H., et al. (2018). Autophagy is essential for the maintenance of amino acids and ATP levels during acute amino acid starvation in MDAMB231 cells. Cell Biochem. Funct. 36, 65-79. doi: 10.1002/cbf.3318

Wagner, M., and Moore, D. D. (2011). Endoplasmic reticulum stress and glucose homeostasis. Curr. Opin. Clin. Nutr. Metab. Care14, 367-373. doi: 10.1097/ MCO.0b013e32834778d4

Wang, H. J., Park, J. Y., Kwon, O., Choe, E. Y., Kim, C. H., Hur, K. Y., et al. (2015). Chronic HMGCR/HMG-CoA reductase inhibitor treatment contributes to dysglycemia by upregulating hepatic gluconeogenesis through autophagy induction. Autophagy 11, 2089-2101. doi: 10.1080/15548627.2015. 1091139

Wang, J., Han, S.-L., Li, L.-Y., Lu, D.-L., Limbu, S. M., Li, D.-L., et al. (2018). Lipophagy is essential for lipid metabolism in fish. Sci. Bull. 63, 879-882. doi: 10.1016/j.scib.2018.05.026

Wang, S., and Kaufman, R. J. (2014). How does protein misfolding in the endoplasmic reticulum affect lipid metabolism in the liver? Curr. Opin. Lipidol. 25, 125-132. doi: 10.1097/MOL.0000000000000056

Wei, C.-C., Luo, Z., Hogstrand, C., Xu, Y.-H., Wu, L.-X., Chen, Y.-X., et al. (2018). Zinc reduces hepatic lipid deposition and activates lipophagy via 
Zn2+/MTF-1/PPAR $\alpha$ and Ca2+/CaMKK $\beta /$ AMPK pathways. FASEB J. doi: 10. 1096/fj.201800463 [Epub ahead of print].

Wei, C.-C., Luo, Z., Song, Y.-F., Pan, Y.-X., Wu, K., and You, W.-J. (2017). Identification of autophagy related genes LC3 and ATG4 from yellow catfish Pelteobagrus fulvidraco and their transcriptional responses to waterborne and dietborne zinc exposure. Chemosphere 175, 228-238. doi: 10.1016/j. chemosphere.2017.02.042

Welte, M. A. (2015). Expanding roles for lipid droplets. Curr. Biol. 25, R470-R481. doi: 10.1016/j.cub.2015.04.004

Xing, H., Wang, Z., Gao, X., Chen, D., Wang, L., Li, S., et al. (2015). Atrazine and chlorpyrifos exposure induces liver autophagic response in common carp. Ecotoxicol. Environ. Saf. 113, 52-58. doi: 10.1016/j.ecoenv.2014. 11.027

Yabu, T., Imamura, S., Mizusawa, N., Touhata, K., and Yamashita, M. (2012). Induction of autophagy by amino acid starvation in fish cells. Mar. Biotechnol. 14, 491-501. doi: 10.1007/s10126-012-9432-9

Yang, L., Li, P., Fu, S., Calay, E. S., and Hotamisligil, G. S. (2010). Defective hepatic autophagy in obesity promotes ER stress and causes insulin resistance. Cell Metab. 11, 467-478. doi: 10.1016/j.cmet.2010.04.005

Yang, Z., and Klionsky, D. J. (2010). Mammalian autophagy: core molecular machinery and signaling regulation. Curr. Opin. Cell Biol. 22, 124-131. doi: 10.1016/j.ceb.2009.11.014

Yu, X., and Long, Y. C. (2014). Autophagy modulates amino acid signaling network in myotubes: differential effects on mTORC1 pathway and the integrated stress response. FASEB J. 29, 394-407. doi: 10.1096/fj.14-25 2841

Yuan, N., Song, L., Zhang, S., Lin, W., Cao, Y., Xu, F., et al. (2015). Bafilomycin A1 targets both autophagy and apoptosis pathways in pediatric B-cell acute lymphoblastic leukemia. Haematologica 100, 345-356. doi: 10.3324/haematol. 2014.113324

Zhang, X., Yuan, Y., Jiang, L., Zhang, J., Gao, J., Shen, Z., et al. (2014). Endoplasmic reticulum stress induced by tunicamycin and thapsigargin protects against transient ischemic brain injury. Autophagy 10, 1801-1813. doi: 10.4161/auto. 32136

Zhou, H., and Liu, R. (2014). ER stress and hepatic lipid metabolism. Front. Genet. 5:112. doi: $10.3389 /$ fgene. 2014.00112

Conflict of Interest Statement: The authors declare that the research was conducted in the absence of any commercial or financial relationships that could be construed as a potential conflict of interest.

Copyright $\odot 2019$ Séité, Pioche, Ory, Plagnes-Juan, Panserat and Seiliez. This is an open-access article distributed under the terms of the Creative Commons Attribution License (CC BY). The use, distribution or reproduction in other forums is permitted, provided the original author(s) and the copyright owner(s) are credited and that the original publication in this journal is cited, in accordance with accepted academic practice. No use, distribution or reproduction is permitted which does not comply with these terms. 